\section{On the Stabilization of a Cable with a Tip Mass}

Ömer Morgül, Bo Peng Rao, and Francis Conrad

Abstract - In this note, we consider a vertical cable which is pinched at the upper end. A mass is attached at the lower end where a control force is also applied. We show that this hybrid system is uniformly stabilized by choosing a suitable control law for the control force depending on the velocity and angular velocity at the free end. Moreover for specific values of the feedback coefficients, we obtain the rate of decay of the energy of the system.

\section{INTRODUCTION}

In this paper, we study the stability of a pinched cable with a tip mass at the free end

$$
\begin{gathered}
u_{t t}-u_{x x}=0 \quad 0<x<1 \quad t>0, \\
u(0, t)=0 \quad t>0, \\
u_{x}(1, t)+m u_{t t}(1, t)=-f(t) \quad t>0
\end{gathered}
$$

where, for simplicity, the wave speed and the length of the cable are chosen to be unity and a subscript letter denotes a partial differential with respect to the corresponding variable. In the above formulation, (1) is the wave equation for the cable, (2) is the boundary condition at the clamped end, (3) is the boundary condition at the free end, $m>0$ is the tip mass, and $f(t)$ is the boundary control force applied at the free end. Our problem is to find a feedback control law $f(t)$ so that the energy of the resulting closed-loop system decays uniformly to zero, both for the cable deflection and the motion of the tip mass.

Recall that the following linear feedback control law

$$
f(t)=\alpha u_{t}(1, t) \quad \alpha>0
$$

has been shown sufficient for the strong stability, but not sufficient for the uniform stability (see [5]). Moreover it was shown in d'Andrea et al. [1], [2], that the system (1)-(3) is strongly stabilizable for the Neuman condition at the end $x=0$ by means of a suitable nonlinear feedback law for $f(t)$, and one can also prove that it is not uniformly stabilized by that feedback.

The same phenomenon of lack of uniform stability occurs also in the case of an elastic beam with a tip mass, called SCOLE model (see [6]). In fact, as shown in Rao [8], we know that the classical feedback that one considers for the standard control problems are a compact perturbation of the uncontrolled system. It is well known that compact controls are not sufficient to provide uniform stability (see [12], [4], and [13]). Hence, to obtain uniform stability, one has to choose "stronger" feedback terms for $f(t)$ such as $u_{x t}(1, t)$ instead of $u_{t}(1, t)$. Consequently, one has to work in higher energy space, and one can establish the uniform decay of energy for any smooth initial data $\left(y(0), y_{i}(0)\right) \in H^{2}(0,1) \times H^{1}(0,1)$. Another way for obtaining uniform stability is to put a feedback control at the end where there is no tip mass (see [9], [10]).

Manuscript received July 30, 1993

O. Morguil is with the Department of Electrical and Electronics Engineering, Bilkent University, 06533, Bilkent, Ankara, Turkey.

B. P. Rao and F. Conrad are with the Département de Mathématiques, Université Nancy 1 , U. A. CNRS 750, INRIA Lorraine Projet Numath, B.P.

239, 54506 Vandoeuvre-lès Nancy, France.

IEEE Log Number 9403954.
The goal of this paper is to investigate the uniform stability of the system (1)-(3) for any initial data $\left(y(0), y_{t}(0)\right) \in H^{1}(0,1) \times$ $L^{2}(0,1)$. This note is organized as follows. In Section II, we establish the uniform decay of the cable deflection by means of an energy method. In Section III, we carry out an analysis of the spectrum, and we give the decay rates for specific cases.

\section{STABILITY RESULTS}

Throughout this paper, we propose the following linear feedback control law $f(t)$

$$
f(t)=a u_{x t}(1, t)+\alpha u_{t}(1, t) \quad t>0
$$

where $a, \alpha$ are positive constants. Upon substituting (5) into (3), the latter becomes

$u_{x}(1, t)+m u_{t t}(1, t)+a u_{x t}(1, t)$

$$
+\alpha u_{t}(1, t)=0 \quad t>0 .
$$

Let $u$ be a smooth solution of the system (1), (2), and (6). We define the auxiliary functions

$$
v(x, t)=u_{t}(x, t), \quad \eta(t)=a u_{x}(1, t)+m u_{t}(1, t) .
$$

Hence we can write formally the system (1), (2), and (6) into the form

$$
\left(\begin{array}{c}
u_{t} \\
v_{t} \\
\eta_{t}
\end{array}\right)=\left(\begin{array}{c}
v \\
u_{x x} \\
-\left(u_{x}(1, t)+\alpha v(1, t)\right)
\end{array}\right) .
$$

Now let us introduce the following spaces

$$
\begin{gathered}
V=\left\{u \in H^{1}(0,1), u(0)=0\right\}, \\
H=\left\{y=(u, v, \eta) \in V \times L^{2}(0,1) \times \mathbf{R}\right\} .
\end{gathered}
$$

For all $y=(u, v, \eta) \in H$ and $\tilde{y}=(\tilde{u}, \tilde{v}, \tilde{\eta}) \in H$, we define the inner product

$$
\langle y, \tilde{y}\rangle_{H}=\int_{0}^{1}\left(u_{x} \tilde{u}_{x}+v \tilde{v}\right) d x+\frac{1}{m+\alpha a} \eta \tilde{\eta} .
$$

Next, we define the unbounded operator $A: D(A) \subset H \rightarrow H$ as

$$
\begin{aligned}
& D(A)=\left\{y=(u, v, \eta) \in\left(H^{2}(0,1) \cap V\right) \times V \times \mathbf{R}\right. \\
& \left.\eta=a u_{x}(1)+m v(1)\right\} \\
& A\left(\begin{array}{l}
u \\
v \\
\eta
\end{array}\right)=\left(\begin{array}{c}
v \\
u_{x x} \\
-\left(u_{x}(1)+\alpha v(1)\right)
\end{array}\right), \quad(u, v, \eta) \in D(A) .
\end{aligned}
$$

With the previous notations, we can formulate system (8) into the abstract form

$$
y_{t}=A y \quad y(0)=y_{0} \in H
$$


Theorem 1: The operator $A: D(A) \subset H \rightarrow H$ defined by (12) and (13) generates a $C_{0}$ semigroup of contractions $T(t)$ on the energy space $H$.

Proof: By the Lumer-Phillips Theorem, it is sufficient to prove that $A$ is an $m$-dissipative operator (see, e.g. [7, p. 14]).

First, any $y=(u, v, \eta) \in D(A)$. a straightforward computation gives

$$
\begin{aligned}
\langle A y \cdot y\rangle_{H}= & \int_{0}^{1}\left(u_{x} v_{x}+v u_{x x}\right) d x \\
& -\frac{1}{m+\alpha a}\left(a u_{x}(1)+m v^{\prime}(1)\right)\left(u_{x}(1)+\alpha v(1)\right) \\
= & -\frac{1}{m+\alpha a}\left(a u_{x}^{2}(1)+\alpha m v^{2}(1)\right)
\end{aligned}
$$

which proves that $A$ is dissipative.

Next for any given $y_{0}=\left(u_{0} \cdot \imath_{0}, \eta_{0}\right) \in H$. we want to solve the system

$$
(I-A) y=y_{0}
$$

which is equivalent to the equations

$$
\begin{gathered}
u-v=u_{0} . \\
\imath-u_{x x}=v_{0} . \\
\eta+u_{x}(1)+\alpha v(1)=\eta_{0} .
\end{gathered}
$$

After obvious eliminations, we obtain the equations

$$
\begin{gathered}
u-u_{x x}=u_{0}+v_{0} \in L^{2}(0.1), \\
u(0)=0 . \\
(1+a) u_{s}(1)+(a+m) u(1)=\eta_{0}+(a+m) u_{0}(1) .
\end{gathered}
$$

Equations (20)-(22) have a unique solution $u \in H^{2}(0,1) \cap V$, which implies that $v=u-u_{0} \in I$. Then $\eta$ can be found from (19). Obviously by construction, $(u v \eta) \in D(A)$. The proof of Theorem 1 now follows from Lumer-Phillips Theorem.

Remark 1: Given $y_{0} \in D\left(A^{2}\right)$, we define $y(t)=T(t) y_{0}=$ $\left(u(t), u^{\prime}(t), \eta(t)\right)$. By the Hille-Yosida Theorem we know that the function $u(t)$ has the smoothness

$$
u(t) \in C^{2}\left(R^{+}: V\right) \cap C^{1}\left(R^{+}: V \cap H^{2}(0,1)\right) .
$$

So for all $y_{0} \in D\left(A^{2}\right)$, we have the equivalence between the system (1), (2), and (6) and system (14). But for general initial data $y_{0} \in H$ or even for $y_{0} \in D(A)$. system (14) is only a weak form of the system (1), (2), and (6). For $y_{0} \in D(A)$, however, $T(t) y_{0}$ is a strong solution of (14).

Now we prove that $T(t)$ decays exponentially uniformly to zero.

Theorem 2: Let $T(t)$ be the $C_{0}$ semigroup of contractions generated by the operator $A$ on the space $H$. Then there exist constants $M>0, \delta>0$ such that

$$
\|T(t)\|_{H} \leq M e^{--\delta t} \quad t \geq 0
$$

Proof: We first define the function

$$
V(t)=t E(t)+2 \int_{0}^{1} x u_{t} u_{x} d x
$$

where the energy $E(t)$ is defined by

$$
\begin{aligned}
E(t) & =\frac{1}{2}\|y(t)\|_{H}^{2} \\
& =\frac{1}{2} \int_{0}^{1}\left(u_{i}^{2}+u_{x}^{2}\right) d x+\frac{1}{2(m+\alpha a)} \eta^{2} .
\end{aligned}
$$

Assume that $y_{0} \in D(A)$, hence $y(t) \in D(A)$ for $t \geq 0$. Then, in view of (15) we have

$$
\begin{aligned}
E_{t}(t) & =\langle y(t), A y(t)\rangle_{H} \\
& =-\frac{1}{m+\alpha a}\left(a u_{x}^{2}(1, t)+\alpha m u_{t}^{2}(1, t)\right) .
\end{aligned}
$$

Next by using Cauchy-Schwartz inequality, it can be easily shown that the following holds

$$
(t-2) E(t) \leq V(t) \leq(t+2) E(t) .
$$

Now differentiating (25) and using (1) we obtain

$$
V_{t}(t)=E(t)+t E_{t}(t)+2 \int_{0}^{1} x\left(u_{x x} u_{x}+u_{t} u_{x t}\right) d x .
$$

Integrating by parts we obtain

$$
\begin{aligned}
& 2 \int_{0}^{1} x\left(u_{x x} u_{x}+u_{x t} u_{t}\right) d x \\
& =u_{x}^{2}(1, t)+u_{t}^{2}(1, t)-\int_{0}^{1}\left(u_{x}^{2}+u_{t}^{2}\right) d x .
\end{aligned}
$$

On the other hand, from (7) it follows that

$$
\eta^{2} \leq 2 a^{2} u_{x}^{2}(1, t)+2 m^{2} u_{t}^{2}(1, t) .
$$

Using (26), (27), (30), and (31) in (29), the latter becomes

$$
\begin{aligned}
V_{t}(t) \leq & -\frac{1}{2} \int_{0}^{1}\left(u_{t}^{2}+u_{x}^{2}\right) d x \\
& -\frac{1}{m+\alpha a}\left(a t-a^{2}-\alpha a-m\right) u_{x}^{2}(1, t) \\
& -\frac{1}{m+\alpha a}\left(\alpha m t-m^{2}-m-\alpha a\right) u_{t}^{2}(1, t) .
\end{aligned}
$$

Thus there exists a constant $T>0$, depending only on the constants $\alpha, m, a$ such that the following holds

$$
V_{t}(t) \leq 0, \quad t \geq T
$$

From (28) and (33) it follows that

$$
E(t) \leq \frac{T+2}{t-2} E(0) \quad t>\max (2, T) .
$$

We deduce that there exist constants $t_{0}>0,0<\gamma<1$ such that

$$
\left\|T\left(t_{0}\right) y_{0}\right\|_{H} \leq \gamma\left\|y_{0}\right\|_{H}
$$

for all $y_{0} \in D(A)$. By the density of $D(A)$ in $H$ it follows that (35) holds for all $y_{0} \in H$. 
Remark 2: Estimate (24) means that the cable deflection decays exponentially uniformly to zero, but this is not necessarily the case for the motion of the tip mass. In fact we know only the exponential decay of the linear combination: $\eta(t)=a u_{x}(1, t)+m u_{t}(1 . t)$ of the cable tension $u_{x}(1 . t)$ and the tip mass velocity $u_{t}(1, t)$.

If we assume that $y_{0} \in D(A)$. then we have also for the graph norm

$$
\left\|T(t) y_{0}\right\|_{D(A)} \leq M e^{-\delta t}\left\|y_{0}\right\|_{D(A)} \text {. }
$$

On the other hand, by Sobolev embedding, we deduce that

$$
u_{t}^{2}(1 . t)+u_{x}^{2}(1 . t) \leq \int_{0}^{1}\left(u_{x l}^{2}+u_{x}^{2}+u_{x x}^{2}\right) d x .
$$

Thus we obtain the exponential uniform decay of the motion of the tip mass for all smooth initial data $y_{0} \in D(A)$.

\section{ANALYSIS OF THE SPECTRUM AND RATE OF DECAY}

In this section we calculate the spectrum of the operator $A$ for special cases, and we prove that the spectrum determines the exponential decay rate given in formula (24) for the cases considered. Here we have to work in the complex Hilbert space of $L^{2}(0,1), V$ and $H$. For convenience, we do not change the notations for these spaces.

Let $\lambda \in \mathrm{C}$ be an eigenvalue, and let $z=(\varphi \vee \eta) \in D(A)$ be an associated eigenvector of $A$. Hence we have

$$
(\lambda I-A) z=0 .
$$

This is equivalent to the following set of equations

$$
\begin{gathered}
\lambda \varphi-\psi=0, \\
\lambda \psi-\varphi_{x x}=0, \\
\lambda \eta+\varphi_{x}(1)+\alpha \psi(1)=0 .
\end{gathered}
$$

By using (39) in (40) we obtain

$$
\lambda^{2} \varphi-\varphi_{x x}=0
$$

whose solutions satisfying $\varphi(0)=0$ are proportional to

$$
\varphi(x)=\sinh \lambda x .
$$

Upon substituting (43) in (39) we obtain

$$
\dot{\psi}(x)=\lambda \sinh \lambda x \text {. }
$$

The last component $\eta$ can be found either by using (41) or by using (12), both yielding the same result. Using the latter we obtain

$$
\eta=\lambda(a \cosh \lambda+m \sinh \lambda) .
$$

By substituting (45) in (41) we obtain the following equation

$$
\lambda[(a \lambda+1) \cosh \lambda+(m \lambda+\alpha) \sinh \lambda]=0 .
$$

Note that $\lambda=0$ cannot be an eigenvalue. For otherwise (43), (44), and (45) imply $\vartheta(x)=\varphi(x)=0$ and $\eta=0$. Hence, all the eigenvalues of the operator $A$ can be found from the equation

$$
(a \lambda+1) \cosh \lambda+(m \lambda+\alpha) \sinh \lambda=0
$$

or equivalently by

$$
((1-a)+(a-m) \lambda) e^{-2 \lambda}+(1+a)+(a+m) \lambda=0 .
$$

If $\alpha \neq 1$ or $m \neq a$, using the Hadamard factorization theorem (see e.g., [3, p. 154]), we can prove that the (48) has an infinite number of roots labeled by $\lambda_{n}$ for $n \in \mathbf{Z}$, which appear in conjugate pairs and $\left|\lambda_{n}\right|$ goes to infinity as $|n|$ goes to infinity. All the eigenvalues $\lambda_{n}$ have geometric multiplicity equal to one.

Now we discuss different situations, including the case $\alpha=1$ and $m=a$.
A. Case $\alpha \neq(\mathrm{m} / \mathrm{a})$

If $(1+\alpha)+(\alpha+m) \lambda_{n}=0$, then by (48), we have also $(1-\alpha)+(a-m) \lambda_{n}=0$, which implies that $\alpha=(m / a)$, a contradiction. Hence we see that (48) is equivalent to

$$
e^{2 \lambda_{n}}=-\frac{(1-\alpha)+(a-m) \lambda_{n}}{(1+\alpha)+(a+m) \lambda_{n}}=-\rho\left(\lambda_{n}\right) .
$$

Taking the logarithms of both sides of (49), we get

$$
\lambda_{n}=\frac{1}{2} \ln \left|\rho\left(\lambda_{n}\right)\right|+\frac{i}{2}\left(\pi+\arg \rho\left(\lambda_{n}\right)\right) \quad(\bmod \pi) .
$$

Assume first that $m \neq a$. Then we have

$$
\lim _{n \rightarrow \infty} \operatorname{Re}\left(\lambda_{n}\right)=\frac{1}{2} \ln \left|\frac{a-m}{a+m}\right| .
$$

In other words, the spectrum has, in that case, a vertical asymptote in the left-half plane. It should be interesting to locate the eigenvalues in the left or the right part of the asymptote. Numerical computations show that there are indeed eigenvalues in the right part of the asymptote.

In the case $m=a$ and $\alpha \neq 1$, (49) reduces to

$$
e^{2 \lambda_{n}}\left(1+\alpha+2 a \lambda_{n}\right)=\alpha-1 \text {. }
$$

For $\alpha>1,(52)$ has a real negative root whereas $\lim _{n \rightarrow \infty} \operatorname{Re}\left(\lambda_{n}\right)=$ $-\infty$. So in these cases, it seems not easy to get the rate of decay of the energy in a simple way from formula (51). This negative result is also conjectured for $\alpha<1$.

On the other hand, we shall now prove that when $\alpha=(\mathrm{m} / \mathrm{a})$, we get indeed the rate of decay of energy.

B. Case $\alpha=(m / a), \alpha \neq 1$

In that case, the eigenvalue equation (47) becomes

$$
(a \lambda+1)(\cosh \lambda+\alpha \sinh \lambda)=0 \text {. }
$$

For the root $\lambda_{*}=-(1 / a)$, the corresponding (not normalized) eigenvector $F_{*}$ is taken as

$$
F_{*}=\left(\begin{array}{c}
\frac{1}{\lambda_{*}} \sinh \lambda_{*} x \\
\sinh \lambda_{*} x \\
a\left(\cosh \lambda_{*}+\alpha \sinh \lambda_{*}\right)
\end{array}\right) .
$$

The remaining eigenvalues are given by the equation

$$
\cosh \lambda+\alpha \sinh \lambda=0
$$

or else

$$
e^{-2 \lambda}(1-\alpha)+1+\alpha=0
$$

which gives

$$
\begin{gathered}
\lambda_{n}=\frac{1}{2} \ln \left|\frac{1-\alpha}{1+\alpha}\right|+\frac{2 n+1}{2} \pi_{i} \quad n \in \mathbf{Z} \quad 0<\alpha<1, \\
\lambda_{n}=\frac{1}{2} \ln \left|\frac{1-\alpha}{1+\alpha}\right|+n \pi i \quad n \in \mathbf{Z} \quad \alpha>1 .
\end{gathered}
$$

In both cases, the corresponding (not normalized) eigenvectors $F_{n}$ are taken as

$$
F_{n}=\left(\begin{array}{c}
\frac{1}{\lambda_{n}} \sinh \lambda_{n} x \\
\sinh \lambda_{n} x \\
0
\end{array}\right) .
$$

Notice that all the roots of (53) have order of multiplicity one, except when $\cosh \lambda_{*}+\alpha \sinh \lambda_{*}=0$. Then $\lambda_{*}$ is a zero of order of two of (53), the other zeros being again of order one. This case has to be studied separately. 
1) Case $\alpha=(m / a) . \alpha \neq 1 \cdot \cosh \lambda_{*}+\alpha \sinh \lambda_{*} \neq 0$ : It is easy to show that the equation $(\lambda I-A) G=F$, with $(\lambda, F)=\left(\lambda_{n}, F_{n}\right)$ or $\left(\lambda_{*} . F_{*}\right)$ has no solution. This means that all the eigenvalues of $A$ have algebraic multiplicity one.

Theorem 3: Assume that $\alpha=(m / a), \alpha \neq 1$ and $\cosh \lambda_{*}+$ $a \sinh \lambda_{*} \neq 0$. Then

i) the system $\left\{F_{*}, F_{n}, n \in \mathbf{Z}\right\}$ is a Riesz basis of $H$,

ii) the uniform decay (24) is valid with the decay rate $\delta$ given by

$$
\delta=\min \left\{\frac{1}{a}, \quad-\frac{1}{2} \ln \left|\frac{1-\alpha}{1+\alpha}\right|\right\} .
$$

Proof:

i) Let $z=\left(\begin{array}{lll}u & v & \eta\end{array}\right) \in H$. We set

$$
c_{*}=\frac{\eta}{a\left(\cosh \lambda_{*}+\alpha \sinh \lambda_{*}\right)} \text {. }
$$

Then

$$
z=\left(\begin{array}{l}
\tilde{u} \\
\hat{v} \\
0
\end{array}\right)+c_{*} F_{*}
$$

where

$$
\begin{aligned}
& \tilde{u}=u+\frac{\sinh \lambda_{*} x}{\left(\cosh \lambda_{*}+\alpha \sinh \lambda_{*}\right)} \eta, \\
& \dot{v}=v+\frac{\sinh \lambda_{*} x}{\cosh \lambda_{*}+\alpha \sinh \lambda_{*}} \lambda_{*} \eta .
\end{aligned}
$$

From [11, Theorem 2.3.1], it follows that

$$
\dot{F}_{n}=\left(\begin{array}{c}
\frac{1}{\lambda_{n}} \sinh \lambda_{n} x^{n} \\
\sinh \lambda_{n} x
\end{array}\right)_{n \in \mathbf{Z}}
$$

in a Riesz basis of $V \times L^{2}(0,1)$. Thus we can write

$$
\left(\begin{array}{l}
\tilde{u} \\
\tilde{v}
\end{array}\right)=\sum_{n \in \mathbf{Z}} c_{n} \dot{F}_{n}
$$

where $c_{n} \in l^{2}(\mathbf{Z})$, and there exist positive constants $C_{1}, C_{2}$ such that

$$
C_{1} \sum_{n \in \mathbf{Z}}\left|c_{n}\right|^{2} \leq\left\|\left(\begin{array}{l}
\tilde{u} \\
\tilde{v}
\end{array}\right)\right\|_{V \times L^{2}}^{2} \leq C_{2} \sum_{n \in \mathbf{Z}}\left|c_{n}\right|^{2}
$$

where we set

$$
\left\|\left(\begin{array}{l}
\tilde{u} \\
\tilde{v}
\end{array}\right)\right\|_{V \times L^{2}}^{2}=\int_{0}^{1}\left(\tilde{u}_{x}^{2}+\tilde{i}^{2}\right) d x .
$$

Now we define

$$
G(x)=\frac{\sinh \lambda_{*} x}{\cosh \lambda_{*}+\alpha \sinh \lambda_{*}} \eta .
$$

Then we have

$$
\begin{aligned}
\|z\|_{H}^{2}= & \int_{0}^{1}\left(\tilde{u}_{x}^{2}+\tilde{v}^{2}-2 G_{x} \tilde{u}_{x}-2 \lambda_{*} G \tilde{v}+G_{x}^{2}+\lambda_{*}^{2} G^{2}\right) d x \\
& +\frac{1}{2 m} \eta^{2}=\left\|\left(\begin{array}{c}
\tilde{u} \\
\tilde{v}
\end{array}\right)\right\|_{V \times L^{2}}^{2}+\frac{1}{2 m} \eta^{2}+M \eta^{2}+N
\end{aligned}
$$

where

$$
M \eta^{2}=\int_{0}^{1}\left(G_{s}^{2}+\lambda_{*}^{2} G^{2}\right) d x
$$

and $M$ is a constant depending only on $a, \alpha$. On the other hand, we set $N=-2 \int_{0}^{1}\left(G_{x} \tilde{u}_{x}+\lambda_{*} G v\right) d x$, so that for any $\sigma>0$, we have

$|N| \leq \sigma \int_{0}^{1}\left(\left|\tilde{u}_{x}\right|^{2}+|\tilde{v}|^{2}\right) d x+\frac{1}{\sigma} \int_{0}^{1}\left(\left|G_{x}\right|^{2}+\lambda_{*}^{2}|G|^{2}\right) d x$.

Using (64) into (65), we get

$$
|N| \leq \sigma \int_{0}^{1}\left(\left|\tilde{u}_{x}\right|^{2}+|\tilde{v}|^{2}\right) d x+\frac{M}{\sigma} \eta^{2} .
$$

Injecting (66) into (63), we deduce that

$$
\begin{aligned}
& \|z\|^{2} \leq(1+\sigma)\left\|\left(\begin{array}{l}
\hat{u} \\
\tilde{v}
\end{array}\right)\right\|_{V \times L^{2}}^{2}+\left(M+\frac{M}{\sigma}+\frac{1}{2 m}\right) \eta^{2}, \\
& \|z\|^{2} \geq(1-\sigma)\left\|\left(\begin{array}{c}
\tilde{u} \\
\dot{v}
\end{array}\right)\right\|_{V \times L^{2}}^{2}+\left(M-\frac{M}{\sigma}+\frac{1}{2 m}\right) \eta^{2} .
\end{aligned}
$$

Next we choose $\sigma>0$ so that

$$
\frac{2 m M}{1+2 m M}<\sigma<1
$$

which implies that

$$
1-\sigma>0, \quad M-\frac{M}{\sigma}+\frac{1}{2 m}>0 .
$$

Since $c_{*}$ is proportional to $\eta$ by $(61)$, combining (67), (68), with (62), we deduce that there exist constants $C_{1}>0, C_{2}>0$ such that

$C_{1}\left(\sum_{n \in Z}\left|c_{n}\right|^{2}+\left|c_{*}\right|^{2}\right) \leq\|z\|_{H}^{2} \leq C_{2}\left(\sum_{n \in Z}\left|c_{n}\right|^{2}+\left|c_{*}\right|^{2}\right)$

which implies that the system $\left\{F_{*}, F_{n}, n \in \mathbf{Z}\right\}$ is a Riesz basis in $H$.

ii) This is an immediate and standard consequence of the fact that the system $\left\{F_{*}, F_{n}, n \in \mathbf{Z}\right\}$ is a Riesz basis in $H$. Moreover we have

$$
\begin{aligned}
\delta & =-\max \left\{\operatorname{Re}\left(\lambda_{n}\right), \operatorname{Re}\left(\lambda_{*}\right), n \in \mathbf{Z}\right\} \\
& =\min \left\{\frac{1}{a} .-\frac{1}{2} \ln \left|\frac{1-\alpha}{1+\alpha}\right|\right\} .
\end{aligned}
$$

2) Case $\alpha=(m / a), \alpha \neq 1, \cosh \lambda_{*}+\alpha \sinh \lambda_{*}=0$ : It is easy to show that if $\cosh \lambda_{*}+\alpha \sinh \lambda_{*}=0$, then $\alpha>1$. Using the same argument in the beginning of the Case 1), one can prove that all the eigenvalues, except $\lambda_{*}=\lambda_{0}$, have algebraic multiplicity one. Whereas the eigenspace relative to $\lambda_{*}$ has dimension one, however, the algebraic multiplicity of $\lambda_{*}$ is exactly two, which is also the order of $\lambda_{*}$ as a zero of (53). Thus the operator $A$ has a "Jordan form." Now

$$
\lambda_{*}=\lambda_{0}=-\frac{1}{a}, \quad \lambda_{n}=\frac{1}{2} \ln \frac{\alpha-1}{\alpha+1}+n \pi i, \quad n \in \mathbf{Z},
$$

$F_{*}=F_{0}=\left(\begin{array}{c}\frac{1}{\lambda_{*}} \sinh \lambda_{*} x \\ \sinh \lambda_{*} x \\ 0\end{array}\right), \quad F_{n}=\left(\begin{array}{c}\frac{1}{\lambda_{n}} \sinh \lambda_{n} x \\ \sinh \lambda_{n} x \\ 0\end{array}\right), \quad n \in \mathbf{Z}$

Clearly, we do not have a basis in $H$. According to the previous remark, we have to add one "generalized eigenvector" of $A$. Recall

$$
D\left(B^{2}\right)=\left(\begin{array}{c}
(u, v, \eta) \in H^{3}(0,1) \times H^{2}(0,1) \times \mathbf{R} ; u(0)=v(0)=u_{x x}(0)=0 \\
\eta=a u_{x}(1)+m v(1), a\left(v_{x}(1)-\lambda_{*} u_{x}(1)\right)+m\left(u_{x x}(1)-\lambda_{*} v(1)\right)=0
\end{array}\right)
$$


that if $B=A-\lambda_{*} I$. then see the equation at the bottom of the previous page and

$$
B^{2}\left(\begin{array}{l}
u \\
v \\
\eta
\end{array}\right)=\left(\begin{array}{c}
u_{x x}-2 \lambda_{*} v+\lambda_{*}^{2} u \\
c_{x x}-2 \lambda_{*} u_{x x}+\lambda_{*}^{2} v \\
0
\end{array}\right) \quad(u, v, \eta) \in D\left(B^{2}\right) .
$$

Thus the equation

$$
B^{2}\left(\begin{array}{l}
0 \\
\hat{\varphi} \\
\eta
\end{array}\right)=0, \quad\left(\begin{array}{l}
0 \\
\varphi \\
\eta
\end{array}\right) \in D\left(B^{2}\right)
$$

reduces to

$$
\begin{gathered}
O_{x x}-2 \lambda_{*} \varphi+\lambda_{*}^{2} \phi=0, \\
\hat{\varphi x x}-2 \lambda_{*} \phi_{x x}+\lambda_{*}^{2} \varphi=0, \\
\phi(0)=\varphi(0)=\phi_{x x}(0)=0, \\
\left(\varphi_{x}(1)-\lambda_{*} \phi_{x}(1)\right)+\alpha\left(\phi_{x x}(1)-\lambda_{*} \varphi(1)\right)=0, \\
\eta=a \phi_{x}(1)+m \varphi(1) .
\end{gathered}
$$

An elementary, but lengthy, computation shows that all the solutions $\phi$ are given by

$$
\phi(x)=c_{1} \sinh \lambda_{*} x+c_{2} x \cosh \lambda_{*} x
$$

where $c_{1}, c_{2}$ are arbitrary constants. Therefore, the kernel of $B^{2}$ is of dimension 2 , with generators

$$
\begin{gathered}
F_{*}=\left(\begin{array}{c}
\frac{1}{\lambda_{*}} \sinh \lambda_{*} x \\
\sinh \lambda_{*} x \\
0
\end{array}\right), \\
F_{* *}=\left(\begin{array}{c}
\frac{1}{\lambda_{*}} x \cosh \lambda_{*} x \\
\frac{1}{\lambda_{*}} \sinh \lambda_{*} x+x \cosh \lambda_{*} x \\
a\left(\sinh \lambda_{*}+\alpha \cosh \lambda_{*}\right)
\end{array}\right) .
\end{gathered}
$$

We observe that the last component of $F_{* *}$ is strictly positive, since $\alpha>1$.

Theorem 4: Assume that $\alpha=(m / a), \alpha \neq 1, \cosh \lambda_{*}+$ $\alpha \sinh \lambda_{*}=0$. Then:

i) the system $\left\{F_{* *}, F_{n}, n \in \mathbf{Z}\right\}$ is a Riesz basis of $H$,

ii) the uniform decay (24) is valid with any

$$
\delta<\frac{1}{a}=-\ln \frac{\alpha-1}{\alpha+1}
$$

Proof:

i) Let $z=\left(\begin{array}{lll}u & v & \eta\end{array}\right) \in H$. We set

$$
c_{* *}=\frac{\eta}{a\left(\sinh \lambda_{*}+\alpha \cosh \lambda_{*}\right)} \text {. }
$$

Then we have

$$
z=\left(\begin{array}{c}
\tilde{u} \\
\tilde{v} \\
0
\end{array}\right)+c_{* *} F_{* *}
$$

where

$$
\begin{gathered}
\tilde{u}=u+\frac{x \cosh \lambda_{*} x}{\sinh \lambda_{*}+\alpha \cosh \lambda_{*}} \eta=u+G_{1} \\
i=v+\frac{\sinh \lambda_{*} x+\lambda_{*} x \cosh \lambda_{*} x}{\sinh \lambda_{*}+\alpha \cosh \lambda_{*}} \eta=v+H_{1} .
\end{gathered}
$$

Then essentially the same proof as for part i) of Theorem 3 works, just replacing $G$ by $G_{1}$ and $\lambda_{*} G$ by $H_{1}$. ii) A straightforward computation shows that the solution of (14) with initial condition $F_{* *}$ is

$$
T(t) F_{* *}=e^{\lambda_{*} t}\left(F_{* *}+t F_{0}\right) .
$$

Due to the presence of the term $t e^{\lambda_{*} t} F_{0}$, this proves that estimate (24) does not hold for $\delta \geq(1 / a)$.

Now let $z \in H$ be an initial condition. Then

$$
z=\sum_{n \in \mathbf{Z}} c_{n} F_{n}+c_{* *} F_{* *}
$$

so that

$$
\begin{aligned}
z(t) & =T(t) z=\sum_{n \in \mathbf{Z}} c_{n} e^{\lambda_{n} t} F_{n}+c_{* *} e^{\lambda_{*} t}\left(F_{* *}+t F_{0}\right) \\
& =\sum_{n \in \mathbf{Z}} c_{n} e^{\lambda_{n} t} F_{n}+\left(c_{0}+c_{* *} t\right) e^{\lambda_{*} t} F_{0}+c_{* *} e^{\lambda_{*} t} F_{* *} .(70)
\end{aligned}
$$

For any $\delta<(1 / a)=\operatorname{Re}\left(\lambda_{n}\right)$, one has

$$
\begin{gathered}
\left|e^{\lambda_{n} t}\right|=e^{-(t / a)} \leq e^{-\delta t}, \\
\left|t e^{\lambda_{*} t}\right| \leq t e^{-(t / a)} \leq \beta(\delta) e^{-\delta t} .
\end{gathered}
$$

Injecting (71) and (72), into (70) and using the property of Riesz basis, we get

$$
\begin{aligned}
\|z(t)\|_{H}^{2} & \leq C_{2}\left(\sum_{n \in \mathbf{Z}^{*}}\left|c_{n} e^{\lambda_{n} t}\right|^{2}+\left|\left(C_{0}+c_{* *} t\right) e^{\lambda_{*} t}\right|^{2}+\left|c_{* *} e^{\lambda_{*} t}\right|^{2}\right) \\
& \leq C_{2}\left(\sum_{n \in \mathbf{Z}^{*}}\left|c_{n}\right|^{2}+2\left|c_{0}\right|^{2}+\left|c_{* *}\right|^{2}\left(1+2 \beta^{2}\right)\right) e^{-2 \delta t} \\
& \leq 2 C_{2}\left(1+\beta^{2}\right)\left(\sum_{n \in \mathbf{Z}}\left|c_{n}\right|^{2}+\left|c_{* *}\right|^{2}\right) e^{-2 \delta t} \\
& \leq \frac{C_{2}}{C_{1}}\left(1+\beta^{2}\right)\|z\|_{H}^{2} e^{-2 \delta t}
\end{aligned}
$$

which yields the desired result.

$$
\text { C. Case } \alpha=(m / a)=1
$$

In that case, $\lambda_{*}=-(1 / a)$ is the only eigenvalue of the operator $A$ and the Riesz basis disappears. We expect that the decay rate is exactly $(1 / a)$. More specifically, we have the following result.

Theorem 5: Assume that $\alpha=(m / a)=1$. Then for all $y_{0} \in H$, we have

$$
T(t) y_{0}=T(2) y_{0} e^{-(1 / a)(t-2)}, \quad \forall t \geq 2 .
$$

Proof: We start with $y_{0} \in D\left(A^{3}\right)$. In that case, we know that $y(t)=T(t) y_{0}=(u(t), v(t), \eta(t))$ is a smooth solution of the system (1), (2), and (6). In particular, we have

$$
u(t) \in C^{4-j}\left(\mathbf{R}^{+} ; H^{j}(0,1)\right), \quad 0 \leq j \leq 4 .
$$

Let $w(x, t)=u(x, t)+a u_{t}(x, t)$. Then by means of the smoothness property (74), we see that $w$ is a smooth solution of the wave equation

$$
w_{t t}-w_{x x}=0, \quad 0<x<1,
$$

$$
w(0, t)=0, \quad w_{x}(1, t)+w_{t}(1, t)=0 .
$$

We deduce from [11, Theorem 2.3.4.1] that

$$
w(x, t)=u(x, t)+a u_{t}(x, t) \equiv 0, \quad \forall t \geq 2 .
$$

It follows that

$$
u(x, t)=u(x, 2) e^{-(1 / a)(t-2)}, \quad \forall t \geq 2 .
$$


Consequently, we find that

$$
\begin{aligned}
\left(\begin{array}{c}
u(x, t) \\
v(x, t) \\
\eta(t)
\end{array}\right) & =\left(\begin{array}{c}
u(x, 2) \\
-\frac{u(x, 2)}{a} \\
a u_{x}(1,2)-u(1,2)
\end{array}\right) e^{-(1 / a)(t-2)} \\
& =T(2) y_{0} e^{-(1 / a)(t-2)}, \quad \forall t \geq 2 .
\end{aligned}
$$

We have proved Theorem 5 for all smooth initial condition $y_{0} \in$ $D\left(A^{3}\right)$. Now let $y_{0} \in H$. Then by the denseness of $D\left(A^{3}\right)$ in $H$, we can find a sequence $y_{0}^{n} \in D\left(A^{3}\right)$ such that $y_{0}^{n} \rightarrow y_{0}$ in $H$. We have thus

$$
T(t) y_{0}^{n}=T(2) y_{0}^{n} e^{-(1 / a)(t-2)}, \quad \forall t \geq 2 .
$$

Passing to the limit in (76), we establish (73) for all initial condition $y_{0} \in H$.

Finally, if the initial condition $y_{0}$ is taken as an eigenvector corresponding to the only eigenvalue $\lambda_{*}=-(1 / a)$, we obtain

$$
T(t) y_{0}=y_{0} e^{-(1 / a) t}, \quad \forall t \geq 0 .
$$

This means that in (24) the rate $\delta=(1 / a)$ is indeed the decay rate. Moreover we have $M=1$, because of the contraction property of the semigroup $T(t)$. The proof is now complete.

\section{CONCLUSION}

In this paper we studied the stability of a cable with a tip mass. The cable is assumed to be fixed at one end and free at the other end, where a mass is also attached. To stabilize this hybrid system, a boundary control force is applied at the free end. It is well known that standard velocity feedback for the control force [see (4)], which is widely used in boundary control systems, yields only strong, but not exponential stability (see e.g., [5]). In this paper we proposed a new control law [see (5)] which contains stronger feedback terms other than the standard velocity feedback term. Such terms are necessary to obtain exponential stability in the closed-loop system, for otherwise the feedback terms may be considered as a compact perturbation of the uncontrolled system which is known to yield only strong, but not exponential stability (see e.g. [1], [2]). We then proved that the system is well posed (Theorem 1) and that the energy associated with the system decays exponentially (Theorem 2 ). It then follows that if the initial data are sufficiently smooth, then both cable deflections and the velocity of the tip mass decay exponentially to zero (Remark 2). We then studied the spectrum of the system for various values of the control parameters and proved that in certain cases the decay rate is related to the spectrum and is determined by the control parameters (Theorems 3, 4, 5).

Part of the results presented in this paper may be extended to the SCOLE system, which consists of a flexible beam with a tip mass (see [6]). This will be the subject of a forthcoming paper.

\section{REFERENCES}

[1] B. d'Andrea-Novel, F. Boustany, and B. P. Rao, "Control of an overhead crane: Feedback stabilization of a hybrid PDE-ODE system," in Proc. First European Contr. Conf., Grenoble, France, July 1991, pp. 314-317.

[2] B. d'Andrea-Novel, F. Boustany, F. Conrad, and B. P. Rao, "Feedback stabilization of a hybrid PDE-ODE system: Application to an overhead crane," Math. Contr. Sig. Syst., to appear.

[3] L. Ash, Complex Variables. New York: Academic Press, 1971.

[4] J. S. Gibson, "A note on stabilization of infinite dimensional linear oscilliators by compact linear feedback," SIAM J. Contr. Optim., vol. 18 , no. 3, pp. 311-316, 1980 .
[5] E. B. Lee and Y. C. You, "Stabilization of a hybrid (string/point mass) system," in Proc. Fifth Int. Conf. Syst. Eng., Dayton, Ohio, Sept. 1987.

[6] W. Littman and L. Markus, "Stabilization of a hybrid system of elasticity by feedback boundary damping," Annali di Matematica Pura ed Applicata, vol. 152, pp. 281-330, 1988.

[7] A. Pazy, Semigroups of Linear Operators and Applications to Partial Differential Equations. New York: Springer-Verlag, 1983.

[8] B. P. Rao, "Stabilization uniforme d'un système hybride en élasticité," C. R. Acad. Sci. Paris, vol. 316, Série I, pp. 261-266, 1993.

[9] _ "Decay estimates of solutions for a hybrid system of flexible structures," European J. Applied Math., vol. 4, pp. 303-319, 1993

[10] _ _ "Uniform stabilization of a hybrid system of elasticity," SIAM J. Contr. Optim., to appear.

[11] P. Rideau, "Contrôle d'un assemblage de poutres flexibles par des capteurs-actionneurs ponctuels: étude du spectre du système," Master's thesis, Ecole Nationale Supérieriure des Mines de Paris, SophiaAntipolis, France, Nov. 1985

[12] D. L. Russell, "Decay rates for weakly damped systems in Hilbert space obtained with control-theoretic models," J. Differential Equations, vol. 19 , pp. $344-370,1975$.

[13] R. Triggiani, "Lack of uniform stabilization for noncontractive semigroups under compact perturbation," in Proc. Am. Math. Soc., vol. 105, pp. 375-383, 1989.

\section{A Remark on Chaotic Behavior in Adaptive Control Systems}

Graciela A. González, María I. Troparevsky, and Carlos E. D'Attellis

Abstract-This note deals with the control of a linear discrete-time system designed on the assumptions that we do not have explicit knowledge of the order of the plant. The objective is to cause the output to track a given constant reference $y^{*}$, when the initial conditions are in a neighborhood of $y^{*}$.

In previous papers the presence of chaos was conjectured for an adaptive scheme where the plant to be controlled was a second-order one undermodeled by a first-order one.

We show that with an adequate choice of the parameter estimator local regulation with exponential convergence of both the output and the parameter is obtained.

\section{INTRODUCTION}

Processes controlled by self-adapting schemes are known to display a rich variety of dynamical features, from global asymptotic stability to chaotic behavior, as reported by Rubio et al. [11] and Salam and Bai [12].

While global convergence of some classes of model reference adaptive control has been established [5] this condition hinges on the knowledge of the order of the system to be controlled. Recently there has been a considerable effort devoted to the analysis of cases where there is a mismatch between the model and the plant, for example unmeasured disturbances as in [2], underestimate of the plant order, or unmodeled nonlinear components as in [17], or poor estimates of the parameters values in the model. Under some circumstances

Manuscript received August 3, 1993; revised December 11, 1993.

G. A. González and M. I. Troparevsky are with the Departamento de Matemática, Facultad de Ciencias Exactas, Universidad de Buenos Aires, Ciudad Universitaria, Pabellón I, 1428-Buenos Aires, Argentina.

C. E. D'Attellis is with the Centro de Cálculo Cientifico (C.A.C.), Comisión Nacional de Energía Atómica, Av. Libertador 8250, 1429-Buenos Aires, Argentina.

IEEE Log Number 9403955 